\title{
Overcoming the Dissipation Condition in Passivity-based Control for a class of mechanical systems
}

\author{
Sergio Delgado*, Paul Kotyczka* \\ * Technische Universität München, Boltzmannstr. 15, D-85748, \\ Garching (Tel: +49-89-289 15679; e-mail: \\ \{s.delgado,kotyczka\}@tum.de). ${ }^{1}$
}

\begin{abstract}
A well-known problem in the application of the Interconnection and Damping Assignment technique for the stabilization of underactuated mechanical systems is dissipation in unactuated coordinates, since it may impede the definiteness requirements for the closed-loop system. Recently, the expansion of the closed-loop Hamiltonian function by a cross term between coordinates and momenta has been explored showing promising results. However, the large number of free parameters is an issue for the tuning of the closed-loop system, and the solution of the matching partial differential equations (PDEs) remains a difficult task. In this work, we aim at giving the closed-loop augmented Hamiltonian more structure in order to simplify the controller parametrization. The result is desired behavior at the equilibrium avoiding the solution of PDEs. Simulations and experiments demonstrate the applicability of the method.
\end{abstract}

\section{INTRODUCTION}

Total energy shaping control techniques like Interconnection and Damping Assignment Passivity-Based control (IDA-PBC) and the method of Controlled Lagrangians (CL) have become popular during the last years. These methods shape the energy of the system but preserve its physical structure and have thus become attractive for the stabilization of (underactuated ${ }^{2}$ ) mechanical systems (see Acosta et al. [2005], Ortega and Spong [2000], Bloch et al. [2000]). The controller design can be summarized in two simple steps: energy shaping of the conservative system in order to assign a local minimum of the closed-loop energy at the desired equilibrium and damping injection to asymptotically stabilize the equilibrium. The role of damping for the stability of mechanical systems is ambiguous, though: it either has a stabilizing or destabilizing effect. Krechetnikov and Marsden [2007] describe with several examples the phenomenon of dissipation-induced instabilities - for systems stabilized by gyroscopic forces. Physical dissipation is mostly neglected in IDA-PBC or CL for the sake of simplicity and mathematical elegance. Yet, it plays a crucial role in the applicability of energy shaping control techniques to real physical systems: as shown in Gómez-Estern and van der Schaft [2004], physical dissipation in unactuated degrees of freedom can impede the implementation of an IDA-PBC controller. The so-called dissipation condition determines if required definiteness properties for the closed-loop system can be fulfilled in the presence of dissipation or not. For a related analysis of the effect of physical damping from the CL point of view see for example Woolsey et al. [2004].

\footnotetext{
1 The authors are with the Institute of Automatic Control (Prof. Boris Lohmann).

2 A mechanical system is said to be underactuated, if not all degrees of freedom can be controlled directly.
}

To overcome the dissipation condition, the desired Hamiltonian function can be augmented by a cross term between coordinates and momenta as reported in Kotyczka and Delgado L. [2012]. This leads to a more general representation of the closed-loop port-Hamiltonian $(\mathrm{pH})$ system and a considerable amount of free parameters. The tuning of the desired system is no longer intuitive: it is not possible to achieve a physically motivated choice of the design parameters, since the approach breaks the physical structure of the system. Transparency with respect to achievable dynamics can however be provided by local linear dynamics assignment presented in Kotyczka [2011]. The resulting nonlinear controller guarantees desired local behavior and provides an estimate of the domain of attraction based on standard IDA-PBC arguments.

In the most common version of IDA-PBC for underactuated mechanical systems, the structure of the interconnection and damping matrices is fixed, and all assignable energy functions are characterized by the solution of a set of PDEs (see e.g. Ortega and Spong [2000]). Here, we show a systematic way to compute the controller without solving any PDEs: we fix the desired Hamiltonian and parametrize the closed-loop system based on the solution of one Lyapunov and some algebraic equations. In Acosta and Astolfi [2009], another approach is pursued to obviate the solution of PDEs for general input-affine systems by designing an approximating integral together with a dynamic extension to replace the PDEs with algebraic inequalities.

The remaining of the paper is organized as follows. Section 2 recalls the main idea of IDA-PBC for underactuated mechanical systems and introduces the dissipation condition. In Section 3 the issues of the dissipation condition are exemplarily explained on a linear mechanical system. Therefrom, an intuitive solution can be derived for the nonlinear system in case the dissipation condition is not satisfied. The main results of this note are presented in 
Section 4 and Section 5. The controller design procedure, simulations and experimental results are finally shown in Section 6 to demonstrate its practicability.

Remark 1. In this paper we only consider the IDA-PBC framework. The CL case can be tackled in a similar manner, since both formulations are equivalent as shown in Blankenstein et al. [2002] and Chang et al. [2002].

Notation: If obvious from the context, arguments are dropped for simplicity. The index 0 as in $x_{0}$ denotes an initial state; in the case of a matrix (e.g. $\left.W_{0}\right)$, it denotes $W(q)$ evaluated at the equilibrium. The column vector of partial derivatives with respect to $x$ is represented as $\nabla_{x}$.

\section{IDA-PBC FOR UNDERACTUATED MECHANICAL SYSTEMS}

Let us first briefly introduce the common IDA-PBC approach for underactuated mechanical systems as presented in Ortega and Spong [2000]. We consider Hamiltonian systems of the form

$$
\left[\begin{array}{c}
\dot{q} \\
\dot{p}
\end{array}\right]=\left[\begin{array}{cc}
0 & I \\
-I & -R(q)
\end{array}\right]\left[\begin{array}{c}
\nabla_{q} H(q, p) \\
\nabla_{p} H(q, p)
\end{array}\right]+\left[\begin{array}{c}
0 \\
G(q)
\end{array}\right] u,
$$

where $q \in \mathbb{R}^{n}$ and $p \in \mathbb{R}^{n}$ are the generalized coordinates and momenta, respectively, $u \in \mathbb{R}^{m}$ is the input, and $G(q) \in \mathbb{R}^{n \times m}$ the input matrix with $\operatorname{rank}(G(q))=m<n$. The dissipation matrix $R(q)=R^{T}(q) \geq 0$ is assumed to satisfy $G_{\perp} R=0^{3}$. The Hamiltonian

$$
H(q, p)=\frac{1}{2} p^{T} M^{-1}(q) p+V(q)
$$

corresponds to the total energy with inertia matrix $M(q)>0$ and potential energy $V(q)$. The state feedback

$$
\begin{array}{r}
u=G^{\dagger}\left(\nabla_{q} H+R M^{-1} p-M_{d} M^{-1} \nabla_{q} H_{d}\right) \\
+G^{\dagger}\left(\left(J_{2}-R_{2}\right) M_{d}^{-1} p\right)
\end{array}
$$

with $G^{\dagger}=\left(G^{T} G\right)^{-1} G^{T}$, transforms (1) into a $\mathrm{pH}$ system

$$
\left[\begin{array}{c}
\dot{q} \\
\dot{p}
\end{array}\right]=\left[\begin{array}{cc}
0 & M^{-1} M_{d} \\
-M_{d} M^{-1} & J_{2}-R_{2}
\end{array}\right]\left[\begin{array}{c}
\nabla_{q} H_{d} \\
\nabla_{p} H_{d}
\end{array}\right]
$$

with new (shaped) energy

$$
H_{d}(q, p)=\frac{1}{2} p^{T} M_{d}^{-1}(q) p+V_{d}(q)
$$

if the projected matching equations

$$
\begin{aligned}
G_{\perp}\left(M_{d} M^{-1} \nabla_{q}\left(p^{T} M_{d}^{-1} p\right)-2 J_{21} M_{d}^{-1} p\right) & \\
-G_{\perp} \nabla_{q}\left(p^{T} M^{-1} p\right) & =0 \\
G_{\perp}\left(\nabla_{q} V-M_{d} M^{-1} \nabla_{q} V_{d}\right) & =0
\end{aligned}
$$

are satisfied. Here, $G_{\perp} \in \mathbb{R}^{(n-m) \times n}$ is a full rank left annihilator satisfying $G_{\perp} G=0$, and $J_{2}=J_{20}(q)+J_{21}(q, p)$ is a skew-symmetric matrix with $J_{21}$ linear in $p^{4}$. The inertia matrix PDE (6) and the potential energy PDE (7) correspond to the terms that are quadratic in $p$ or independent from $p$, respectively. If further

$$
q^{*}=\arg \min V_{d}(q), M_{d}(q)>0 \text { and } R_{2}(q) \geq 0
$$

in a neighborhood of $q^{*}$, then the equilibrium $\left(q^{*}, 0\right)$ is (locally) stable with Lyapunov function $H_{d}(q, p)$. Asymptotic stability can be shown by invoking LaSalle's invariance principle. See Gómez-Estern and van der Schaft [2004], and Ortega and Spong [2000] for proofs and details.

\footnotetext{
3 no dissipation in unactuated coordinates

4 Note that $J_{20}$ is chosen such that $G_{\perp} J_{20}=0$ and $R_{2}=G K_{d} G^{T}$ for a positive semidefinite matrix $K_{d}(q) \in \mathbb{R}^{m \times m}$, since $G_{\perp} R=0$.
}

\subsection{Dissipation Condition}

In contrast to above, we consider the case $G_{\perp} R \neq 0$. Thus, we additionally get a new set of algebraic matching equations (corresponding to the terms that are linear in $p$ )

$$
G_{\perp}\left(R M^{-1} p+\left(J_{20}-R_{2}\right) M_{d}^{-1} p\right)=0 .
$$

Gómez-Estern and van der Schaft [2004] derive from (9) the dissipation condition

$$
G_{\perp}\left(R M^{-1} M_{d}+M_{d} M^{-1} R\right) G_{\perp}^{T} \geq 0,
$$

and show, that it is a necessary and sufficient condition for the existence of a passive closed-loop system with positive definite storage function $H_{d}$. It is yet known, that in the presence of physical damping in unactuated degrees of freedom for many mechanical systems - such as the Acrobot system, the Furuta and the inverted pendulum among others - it is not possible to find a solution of the matching equations (6), (7), and (9) which satisfies the definiteness requirements (8) (see for example Kotyczka and Delgado L. [2012], Gómez-Estern and van der Schaft [2004], or Woolsey et al. [2004] for the CL point of view).

\section{MOTIVATING EXAMPLE}

The idea of the present note is easily motivated by looking at a linear mechanical system. A substantial analysis of IDA-PBC for linear time-invariant (LTI) systems can be found in Prajna et al. [2002] and Ortega and Liu [2012]. The CL case is treated in Zenkov [2002]. We consider LTI mechanical systems represented in Hamiltonian form

$\dot{x}=\left[\begin{array}{c}\dot{q} \\ \dot{p}\end{array}\right]=\left[\begin{array}{cc}0 & I \\ -I & -R\end{array}\right]\left[\begin{array}{c}\nabla_{q} H(q, p) \\ \nabla_{p} H(q, p)\end{array}\right]+\left[\begin{array}{c}0 \\ G\end{array}\right] u=A x+B u$,

where the constant system matrices can be written as:

$$
A=\left[\begin{array}{cc}
0 & M^{-1} \\
-Q & -R M^{-1}
\end{array}\right], B=\left[\begin{array}{c}
0 \\
G
\end{array}\right] .
$$

The Hamiltonian in (11) takes the quadratic form

$$
H(q, p)=\frac{1}{2} p^{T} M^{-1} p+\frac{1}{2} q^{T} Q q .
$$

As in the previous section, the goal is to transform (11) by state feedback into a new LTI mechanical system

$$
\left[\begin{array}{c}
\dot{q} \\
\dot{p}
\end{array}\right]=\left[\begin{array}{cc}
0 & M^{-1} M_{d} \\
-M_{d} M^{-1} & J_{2}-R_{2}
\end{array}\right]\left[\begin{array}{cc}
Q_{d} & 0 \\
0 & M_{d}^{-1}
\end{array}\right]\left[\begin{array}{l}
q \\
p
\end{array}\right]=A_{d} x
$$

with shaped energy function

$$
H_{d}(q, p)=\frac{1}{2} p^{T} M_{d}^{-1} p+\frac{1}{2} q^{T} Q_{d} q
$$

The applicability of IDA-PBC to LTI systems is equivalent to the solvability of the LMIs (Proposition 3.1 in Ortega and Liu [2012] and Proposition 7 in Prajna et al. [2002])

$$
\begin{aligned}
P & >0 \\
\operatorname{sym}\left\{B_{\perp} A P^{-1} B_{\perp}^{T}\right\} & \leq 0,
\end{aligned}
$$

where $B_{\perp}$ is a full rank left annihilator of $B\left(B_{\perp} B=0\right)$. The matrix $P$ is the Hessian of the desired quadratic energy function which in this case has a predefined blockdiagonal structure arising from (15):

$$
P=\left[\begin{array}{cc}
Q_{d} & 0 \\
0 & M_{d}^{-1}
\end{array}\right]
$$

The matching of (11) and (14) is satisfied if there exists a solution of the set of LMIs (16) and (17) restricted to 
(18) (see e.g. Zenkov [2002] and Kotyczka and Delgado L. [2012]). The LMI (17) can also be written as

$$
\begin{array}{r}
G_{\perp}\left(M_{d} M^{-1}-Q Q_{d}^{-1}\right)=0 \\
-G_{\perp}\left(R M^{-1} M_{d}+M_{d} M^{-1} R\right) G_{\perp}^{T} \leq 0,
\end{array}
$$

which represent the matching of the potential energy (7), and the dissipation condition (10), respectively. LTI Systems trivially satisfy the kinetic energy matching equation. For the inverted pendulum, there exist no $M_{d}, Q_{d}>0$ which solve (19) and (20) in the case $G_{\perp} R \neq 0$.

The central question which is addressed in this note is whether or not it is possible to transform a damped mechanical system into a closed-loop $\mathrm{pH}$ system by static feedback if the dissipation condition is not satisfied. Ortega and Liu [2012] showed that IDA-PBC is equivalent to stabilizability. The (damped) inverted pendulum is controllable and thus stabilizable. By allowing off diagonal entries in the matrix $P$ - representing a cross term between coordinates and momenta in the energy function (15) - the set of LMIs (16) and (17) can be easily solved.

Remark 2. In Zenkov [2002], the stabilization of (conservative) linear mechanical systems using only position feedback in the CL framework is discussed. Therein, the closed-loop Hamiltonian is initially assumed to have a nonblock-diagonal Hessian, which corresponds to the structure of the closed-loop Hamiltonian (21) in the present note.

\section{MAIN RESULT}

In Kotyczka and Delgado L. [2012] the violation of the dissipation condition in applying the IDA procedure for mechanical systems has been overcome by assuming a closed-loop Hamiltonian, augmented by a mixed term of coordinates and momenta $p^{T} n(q)$. The approach was motivated by the existence of a stabilizing linear state feedback. The Lyapunov function related to the stabilization problem is shown to possess a non-block-diagonal solution. However, a series of difficulties arises in the application of the proposed augmented IDA-PBC approach: The solution of additional PDEs for the new functions $n_{i}(q)$ is required, the estimate of the region of attraction is poor, and the controller becomes confusingly complicated. The present work aims at solving some of these issues. It turns out that the choice $p^{T} n(q)=-p^{T} K(q) \nabla_{q} V_{d}(q)$ for a regular matrix $K(q)$ simplifies the augmented IDA-PBC approach. Consider the Hamiltonian ${ }^{5}$

$$
H_{d}(q, p)=\frac{1}{2} p^{T} M_{d}^{-1} p+V_{d}(q)-p^{T} K \nabla_{q} V_{d}(q)
$$

for the generalized target $\mathrm{pH}$ system

$$
\mathcal{F} \nabla H_{d}=\left[\begin{array}{cc}
W(q) & X(q) \\
Y(q) & Z(q)+J_{21}(q, p)
\end{array}\right]\left[\begin{array}{c}
\nabla_{q} H_{d}(q, p) \\
\nabla_{p} H_{d}(q, p)
\end{array}\right],
$$

with $J_{21}(q, p)=-J_{21}(q, p)^{T}$ linear in $p$. Note that the closed-loop interconnection and damping matrices in $\mathcal{F}(q, p)=\mathcal{J}(q, p)-\mathcal{R}(q)$ are of a more general form as in the classic approach. The goal is to find a static state feedback which renders (1) the modified $\mathrm{pH}$ system (22). For a given closed-loop Hamiltonian $(21), W(q)$ and $X(q)$ can be explicitly calculated: The matching of the first rows of (1) and (22) leads to - splitting the equation in terms independent and linear in $p$ :

\footnotetext{
5 The matrices $K$ and $M_{d}$ are chosen to be constant for simplicity.
}

$$
\begin{aligned}
W\left(\nabla_{q}^{2} V_{d} K^{T}\right)-X M_{d}^{-1}+M^{-1} & =0 \\
(W-X K) \nabla_{q} V_{d} & =0 .
\end{aligned}
$$

With $V_{d}, M_{d}$ and $K$ fixed, (23) and (24) are satisfied by

$$
\begin{aligned}
X & =M^{-1}\left(M_{d}^{-1}-K \nabla_{q}^{2} V_{d} K^{T}\right)^{-1}, \\
W & =X K=M^{-1}\left(M_{d}^{-1}-K \nabla_{q}^{2} V_{d} K^{T}\right)^{-1} K .
\end{aligned}
$$

Furthermore, the unactuated part of the second rows of (1) and (22) must match. A sufficient condition is the solution of the new matching equations (splitting the equation in different dependencies on $p$ - quadratic, linear and independent):

$$
\begin{gathered}
G_{\perp}\left(Y \nabla_{q}\left(p^{T} M_{d}^{-1} p\right)+2 J_{21} M_{d}^{-1} p\right) \\
+G_{\perp} \nabla_{q}\left(p^{T} M^{-1} p\right)=0 \\
G_{\perp}\left(R M^{-1}+Z M_{d}^{-1}-Y \nabla_{q}^{2} V_{d} K^{T}\right) p \\
-G_{\perp} J_{21} K \nabla_{q} V_{d}=0 \\
G_{\perp}\left(\nabla_{q} V+(Y-Z K) \nabla_{q} V_{d}\right)=0 .
\end{gathered}
$$

Assumption 1. The inertia matrix $M$ does not depend on unactuated coordinates, i.e. $G_{\perp} \nabla_{q}\left(p^{T} M^{-1} p\right)=0$.

Proposition 1. Given the solution $L(q)$ of

$$
G_{\perp}\left(\nabla_{q} V+(L+R) M^{-1} M_{d} K \nabla_{q} V_{d}\right)=0
$$

for a given mechanical system (1) with inertia matrix $M$, potential energy $V$ and dissipation matrix $R$, and for fixed $V_{d}, M_{d}$ and $K$, the state feedback

$$
\begin{aligned}
& \qquad \begin{array}{l}
u=G^{\dagger}\left(\nabla_{q} H+R M^{-1} p+Y \nabla_{q} H_{d}+Z \nabla_{p} H_{d}\right) \\
\text { with } G^{\dagger}=\left(G^{T} G\right)^{-1} G^{T}, Y=L W, \text { and } \\
Z=\left(Y \nabla_{q}^{2} V_{d} K^{T}-R M^{-1}\right) M_{d}+G v^{T},
\end{array}
\end{aligned}
$$

transforms (1) into the closed-loop system (22).

Proof. Let $J_{21}=0$ and $M_{d}$ be constant, such that (27) is satisfied. The matching of the actuated part of (1) and (22) yields the control law (31). The solution of (28) requires

$$
Z=\left(Y \nabla_{q}^{2} V_{d} K^{T}-R M^{-1}\right) M_{d}+G v^{T}
$$

for an arbitrary vector $v=v(q) \in \mathbb{R}^{n}$. With $Z$ as in (32), equation (29) takes the form

$$
G_{\perp}\left(\nabla_{q} V+\Sigma \nabla_{q} V_{d}\right)=0
$$

where $\Sigma=Y-\left(Y \nabla_{q}^{2} V_{d} K^{T}-R M^{-1}\right) M_{d} K$, or after some simple calculations

$$
\begin{aligned}
\Sigma & =\left(Y K^{-1}\left(M_{d}^{-1}-K \nabla_{q}^{2} V_{d} K^{T}\right)+R M^{-1}\right) M_{d} K \\
& =\left(Y W^{-1}+R\right) M^{-1} M_{d} K .
\end{aligned}
$$

according to (26). Defining $L=Y W^{-1}$ and rewriting equation (33) with (34) finishes the proof.

\section{STABILITY AND CONTROLLER DESIGN PROCEDURE}

One of the fundamental difficulties of IDA-PBC is the parametrization of the closed-loop system: For the desired equilibrium to be stable, $\left(q^{*}, 0\right)$ needs to be an isolated minimum of the desired Hamiltonian, and $\mathcal{R}(q) \geq 0$ is required around $q^{*}$. The vast amount of degrees of freedom of the presented approach makes it hard to choose an appropriate parametrization. Stability and desired local behavior for the closed-loop system can be guaranteed, however, by matching the linearized closed-loop $\mathrm{pH}$ system with a desired LTI system. 
Assumption 2. $\left(q^{*}, 0\right)$ is an admissible equilibrium, i.e. $\left.G^{\perp} \nabla_{q} V(q)\right|_{q^{*}}=0$ holds.

Let $\Delta \dot{x}=A \Delta x+B u$ be the linearized mechanical system around the equilibrium $x^{*}=\left(q^{* T}, 0^{T}\right)^{T},(\Delta x=x-$ $\left.x^{*}=\left(q-q^{*}, p\right)\right)$ and let the pair $(A, B)$ be controllable. Design a linear state feedback $u=-D \Delta x$ such that the state matrix $A_{d}=A-B D$ is Hurwitz. Then there exists a unique positive definite matrix $P$, which solves the Lyapunov equation

$$
A_{d} P^{-1}+P^{-1} A_{d}^{T}=-2 R_{0}
$$

for any $R_{0}>0$ (see e.g. Boyd et al. [1994]).

Proposition 2. Let the linear feedback $u=-D \Delta x$ for the damped underactuated mechanical system (1) result in a closed-loop system locally approximated by the state matrix $A_{d}$. Take a positive definite matrix $R_{0}>0$ and compute the solution

$$
P=\left[\begin{array}{ll}
P_{11} & P_{12} \\
P_{12}^{T} & P_{22}
\end{array}\right]>0
$$

of (35). Set

$$
\begin{array}{r}
Q_{d}=P_{11}, \quad M_{d}=P_{22}^{-1}, \quad K=-P_{12}^{T} P_{11}^{-1} \\
\text { and }\left[\begin{array}{cc}
W_{0} & X_{0} \\
Y_{0} & Z_{0}
\end{array}\right]=A_{d} P^{-1} .
\end{array}
$$

Fix $V_{d}(q)$ with $\left.\nabla_{q} V_{d}\right|_{q^{*}}=0$ and $\left.\nabla_{q}^{2} V_{d}\right|_{q^{*}}=Q_{d}$ and suppose one can find a solution $L(q)$ of (30), satisfying $L\left(q^{*}\right)=Y\left(q^{*}\right) W\left(q^{*}\right)^{-1}=Y_{0} W_{0}^{-1}$. Then, the nonlinear control law (31) asymptotically stabilizes the equilibrium $\left(q^{*}, 0\right)$. From the largest bounded level set of $H_{d}(q, p)$ where $\mathcal{R}(q)>0$ holds, an estimate of the domain of attraction can be derived. The closed-loop system is locally approximated by

$$
\Delta \dot{x}=A_{d} \Delta x .
$$

Proof. The control law (31) transforms (1) into a portHamiltonian system (22) under the conditions of the proposition and with $W, X$ and $Z$ according to (25), (26) and (32), respectively. From the parameter choice in (37) and the structure of the closed-loop energy (21),

$$
\left.\frac{\partial^{2} H_{d}}{\partial x^{2}}\right|_{x^{*}}=P>0
$$

can be deduced, i.e. positive definiteness of $H_{d}$ in an open neighborhood of $\left(q^{*}, 0\right)$ is guaranteed. The dissipation matrix at the equilibrium is

$$
\mathcal{R}\left(q^{*}\right)=-\frac{1}{2}\left(A_{d} P^{-1}+P^{-1} A_{d}\right)=R_{0}>0 .
$$

Since the elements of $\mathcal{R}(q)$ are continuous functions in $q$, strong dissipativity in an open neighborhood of $\left(q^{*}, 0\right)$ is guaranteed. An estimate of the region of attraction follows from usual Lyapunov arguments. The linearization of the closed-loop pH system around $\left(q^{*}, 0\right)$ yields directly $(39)$.

Six steps summarize the controller design procedure

Step 1: Linearize the mechanical system around the desired equilibrium $\left(q^{*}, 0\right)$ and design a linear state feedback $u=-D \Delta x$, such that the closed-loop dynamics are given by

$$
\Delta \dot{x}=A_{d} \Delta x, \quad \Delta x=\left(q-q^{*}, p\right) \in \mathbb{R}^{2 n}
$$

with $A_{d}$ Hurwitz. Desired local performance properties can be formulated in terms of the eigenvalues of $A_{d}$ or an LQR design to determine $D$.
Step 2: Fix $R_{0}=R_{0}^{T}>0$ and calculate the solution $P^{-1}$ of the Lyapunov equation

$$
A_{d} P^{-1}+P^{-1} A_{d}^{T}=-2 R_{0} .
$$

The matrix $P$ is the Hessian of the desired Hamiltonian function at the equilibrium:

$$
\left.\nabla^{2} H_{d}(q, p)\right|_{\left(q^{*}, 0\right)}=\left[\begin{array}{cc}
Q_{d} & -Q_{d} K^{T} \\
-K Q_{d} & M_{d}^{-1}
\end{array}\right]=P .
$$

Further, calculate the interconnection and damping matrices at the equilibrium:

$$
\left[\begin{array}{cc}
W_{0} & X_{0} \\
Y_{0} & Z_{0}
\end{array}\right]=A_{d} P^{-1}
$$

Step 3: Fix the potential energy of the closed-loop system $V_{d}(q)$, such that

$$
\left.\nabla_{q}^{2} V_{d}(q)\right|_{q^{*}}=Q_{d},\left.\quad \nabla_{q} V_{d}(q)\right|_{q^{*}}=0
$$

Step 4: Solve

$$
G_{\perp}\left(\nabla_{q} V+(L+R) M^{-1} M_{d} K \nabla_{q} V_{d}\right)=0
$$

for an arbitrary matrix $L$ satisfying $L\left(q^{*}\right)=Y_{0} W_{0}^{-1}$

Step 5: Calculate

$$
Y=L W=L M^{-1}\left(K^{-1} M_{d}^{-1}-\nabla_{q}^{2} V_{d} K^{T}\right)^{-1}
$$

and

$$
Z=\left(Y \nabla_{q}^{2} V_{d} K^{T}-R M^{-1}\right) M_{d}+G v^{T}
$$

with an arbitrary vector $v=v(q)$ such that $Z\left(q^{*}\right)=Z_{0}$.

Step 6: Compute the control law (31)

The approach is quite systematic and therefore easy to implement. Only Step 4 should be done with care: Some elements of $L$ might need to be smartly fixed in order to get a suitable solution. In Step 5, the vector $v$ can be further used to inject nonlinear damping (see e.g. example below). Furthermore, the choice of $R_{0}$ has some implications regarding the estimate of the region of attraction. How to optimally choose $R_{0}$ is still an open question.

\section{EXAMPLE - INVERTED PENDULUM ON A CART}
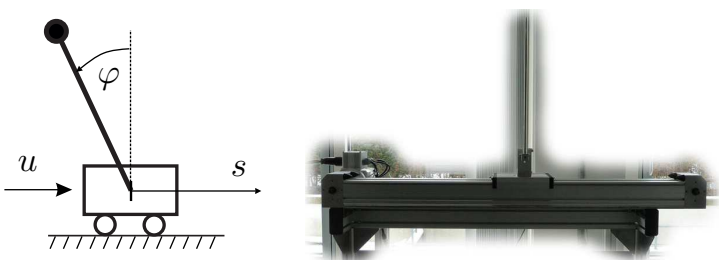

Fig. 1. The inverted pendulum on a cart. Scheme (left) and test rig (right).

To illustrate the approach, consider the pendulum on a cart depicted in Figure 1. This is a classical example of an underactuated mechanical system: it has one single input $u$ and two degrees of freedom corresponding to the horizontal motion of the cart and the rotation of the pendulum, represented in local coordinates by $q=(s, \varphi)^{T}$. The equations of motion after a partial feedback linearization (PFL) (see Spong [1994]) are given by (1) with

$$
\begin{gathered}
M=I_{2 \times 2}, \quad V(\varphi)=a g \cos \varphi, \\
G=\left[\begin{array}{c}
1 \\
a \cos \varphi
\end{array}\right], \quad R=\left[\begin{array}{cc}
0 & 0 \\
0 & r_{\varphi}
\end{array}\right],
\end{gathered}
$$


where $I_{2 \times 2}$ denotes the identity matrix (i.e. $p=\dot{q}$ ), $g$ is the gravity constant and $r_{\varphi}$ the normalized viscous damping coefficient for the unactuated coordinate (the damping in the actuated coordinate is assumed to be compensated). The parameter $a$ is given by

$$
a=\frac{m_{p} l_{p}}{m_{p} l_{p}^{2}+\theta_{p}},
$$

where $l_{p}$ is the distance from the rotation axis to the center of gravity of the pendulum, and $m_{p}$ and $\theta_{p}$ are the pendulum mass and moment of inertia, respectively. The system parameters of the test rig are shown in Table $1^{6}$.

\begin{tabular}{cc} 
Parameter & Value \\
\hline$a$ & 3.9 \\
$g$ & 9.81 \\
$r_{\varphi}$ & $3.34 \cdot 10^{-4}$
\end{tabular}

Table 1. System parameters

\subsection{Controller Design}

The linearized system around the unstable equilibrium is controllable. It is thus possible to design a linear state feedback, such that the equation of motion of the closedloop system is locally approximated by $\dot{x}=A_{d} x$, where $x=(\Delta s, \Delta \varphi, \dot{s}, \dot{\varphi})^{T}$ and $A_{d}$ is Hurwitz. The assumptions above are valid for the inverted pendulum after a PFL: The inertia matrix does not depend on unactuated coordinates, and the desired equilibrium is admissible. We can therefore apply the six steps presented in Section 5 to design a nonlinear stabilizing controller for the system.

The steps 1-3 are implemented using a LQR controller (eigenvalues at $-163.6,-1.03,-6.2,-6.1$ ) and with

$$
R_{0}=\operatorname{diag}\left\{1,1, \frac{1}{3}, \frac{1}{3}\right\} \text { and } V_{d}=\frac{1}{2} q^{T} Q_{d} q .
$$

Fix the elements of the first row of $L$ to the constant values $L(q)_{11}=L\left(q^{*}\right)_{11}$ and $L(q)_{12}=L\left(q^{*}\right)_{12}$ to calculate the solution of (30) (Step 4). Equation (30) results in

$$
\begin{array}{r}
0=\left(c_{1} L_{2,1}+c_{2} \cos \varphi+c_{3} L_{2,2}+c_{4}\right) s+ \\
c_{5} \varphi \cos \varphi+\left(c_{6} L_{2,2}+c_{7} L_{2,1}+c_{8}\right) \varphi+c_{9} \sin \varphi
\end{array}
$$

for some constants $c_{i}$. The remaining two elements $L_{2,1}$ and $L_{2,2}$ are chosen depending only on the angle $\varphi$. The matrices $Y$ and $Z$ are calculated in Step 5 with

$$
v^{T}=v_{0}^{T}+K_{d i}(\varphi) G^{T}
$$

where the constant vector $v_{0}$ is chosen such that $Z\left(q^{*}\right)=$ $Z_{0}$ and $K_{d i}=200\left(\frac{1}{\cos \varphi}-1\right):$ It turns out, that adding more damping for larger values of $\varphi$ improves the transient behavior. Step 6 is straightforward.

\subsection{Simulations}

Figure 2 shows the response of the system controlled with the LQR controller of the previous section, with an IDA-PBC controller as found in Acosta et al. [2005] for the undamped system as an exponent of the classical passivity-based control approach for mechanical systems ${ }^{7}$

6 The system has been non-dimensionalized

7 The IDA-PBC controller has been parametrized with the aim of a large estimate of the region of attraction. Other parametrizations (and approaches) show different responses, the oscillations and the relative slow convergence of the cart's position, however, remain. See e.g. Woolsey et al. [2004] for the CL case. and with the augmented IDA-PBC controller presented in this paper. Near the desired equilibrium, the systems

$$
q_{0}=\left(\begin{array}{c}
0 \\
0.2
\end{array}\right)
$$
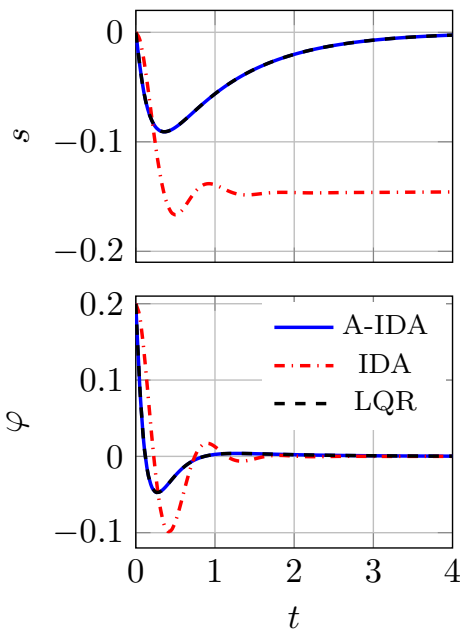

$$
q_{0}=\left(\begin{array}{c}
0 \\
0.95
\end{array}\right)
$$
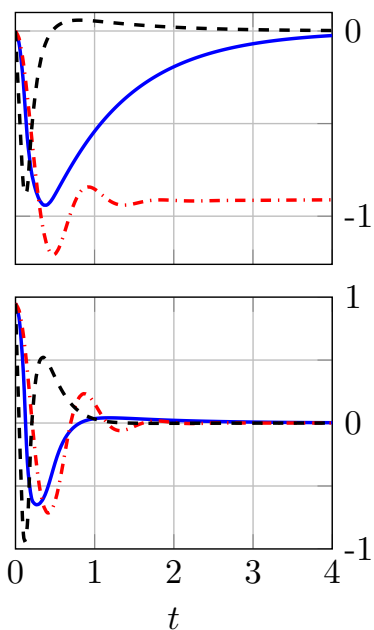

Fig. 2. Response of the cart position $s$ (top) and angle of the pendulum $\varphi$ (bottom) for two different initial angles $\varphi_{0}=0.2 \mathrm{rad}$ (left) and $\varphi_{0}=0.95 \mathrm{rad}$ (right) and $s_{0}=\dot{s}_{0}=\dot{\varphi}_{0}=0$.

controlled with the augmented IDA-PBC and with the LQR controller behave equal. The first one shows, however, a slightly smoother response for larger initial angles. Figure 3 shows level sets of the augmented closed-loop Hamiltonian $H_{d}$ in the $(s, \varphi)$-plane, where $R_{d}>0$. The region bounded by the largest level set is an estimate of the domain of attraction of the equilibrium point: Since the energy function is of quadratic form and the dissipation solely depends on the angle $\varphi$, the 4-dimensional sublevel set of $H_{d}$ completely contained in the region where $R_{d}>0$ is an estimate of the domain of attraction. Table 2 shows

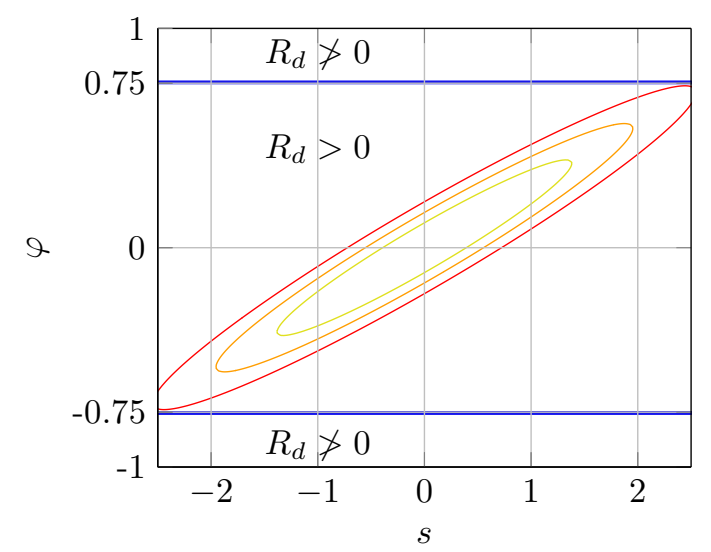

Fig. 3. Level sets of $H_{d}$ in the plane $p=0$ and level set where the smallest eigenvalue of $R_{d}$ equals 0 .

the maximal stabilizable initial angle $\varphi_{0}$ when starting at rest for the different controller types. These values have been determined by simulation ${ }^{8}$.

8 In Woolsey et al. [2004] the equilibrium is asymptotically stable for $\left|\varphi_{0}\right|<\frac{\pi}{2}$ 
Stabilizable initial angles in radians

$\begin{array}{cl}\text { Classic IDA } & -1.45<\varphi_{0}<1.45 \\ \text { Augmented IDA } & -1.20<\varphi_{0}<1.20 \\ \text { LQR } & -0.96<\varphi_{0}<0.96\end{array}$

Table 2. Simulative estimate of the stabilizable initial angles $\varphi_{0}$ for $\left(s_{0}=0, \varphi_{0}, \dot{s}_{0}=0, \dot{\varphi}_{0}=0\right)$

Remark 3. The fact that the dissipation condition is not satisfied does not imply instability of the closed-loop system. It is in fact possible to prove stability for the system controlled by the classical IDA-PBC approach in the presence of physical damping by a spectral stability analysis associated with the linearized dynamics as shown in Woolsey et al. [2004]. However, the analysis is cumbersome and one loses the Lyapunov function, and therewith the proof of the region of attraction. On the other hand, Woolsey et al. [2004] confirm that physical damping degrades the local performance of the energy shaping controller, whereas a (well-tuned) linear static state feedback ensures good local performance eliminating undesired oscillations.

\subsection{Experimental results}

Figure 4 shows the behavior of the augmented IDA-PBC controlled test rig. The same controller parametrization from the simulation has been also used for the experiments, which has been chosen rather "slow" to clearly visualize the results. The desired position of the cart changes from $-0.1 \mathrm{~m}$ to $0.1 \mathrm{~m}$ at $0.85 \mathrm{~s}$. As shown in the plot, the cart smoothly reaches the desired position as expected, keeping the pendulum close to its desired equilibrium and showing a similar transient to that of the simulations.

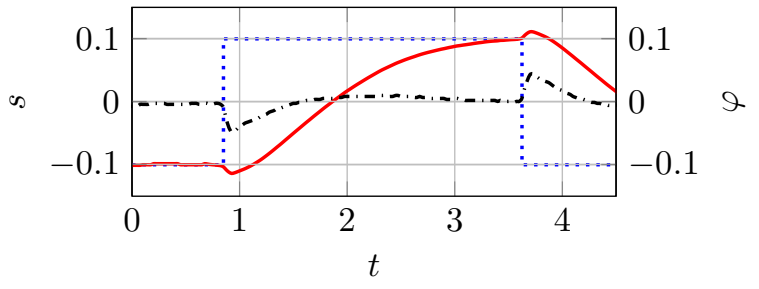

Fig. 4. Position control of the inverted pendulum: the dashdotted line shows the behavior of the pendulum's angle $\varphi$ and the solid line represents the position of the cart $s$

\section{CONCLUSION}

This note presents an IDA-PBC controller design approach for underactuated mechanical systems based on a more general closed-loop Hamiltonian function which a) is easy to parametrize b) does not get affected by physical damping, since dissipation is considered in the controller design and c) does not require the solution of any PDE. A framework of 6 steps has been presented for the controller design for a class of mechanical systems. Simulations and experimental results confirm the applicability of the method.

\section{REFERENCES}

J.A. Acosta and A. Astolfi. On the pdes arising in ida-pbc. In Decision and Control, 2009 held jointly with the 2009 28th Chinese Control Conference. CDC/CCC 2009.
Proceedings of the 48th IEEE Conference on, pages 2132-2137, 2009. doi: 10.1109/CDC.2009.5400580.

J.A. Acosta, R. Ortega, A. Astolfi, and A.D. Mahindrakar. Interconnection and damping assignment passivitybased control of mechanical systems with underactuation degree one. Automatic Control, IEEE Transactions on, 50(12):1936 - 1955, dec. 2005. ISSN 0018-9286. doi: 10.1109/TAC.2005.860292.

G. Blankenstein, R. Ortega, and A.J. Schaft van der. The matching conditions of controlled lagrangians and ida-passivity based control. International Journal of Control, 75(9):645-665, 2002.

A. M. Bloch, D. E. Chang, N. E. Leonard, and J. E. Marsden. Controlled lagrangians and the stabilization of mechanical systems i: the first matching theorem. IEEE Transactions on Automatic Control, 45: 2253-2270, 2000

S. Boyd, L. El Ghaoui, E. Feron, and V. Balakrishnan. Linear Matrix Inequalities in System and Control Theory, volume 15 of Studies in Applied Mathematics. SIAM, Philadelphia, PA, June 1994. ISBN 0-89871-334-X.

D.E. Chang, A. M. Bloch, N. E. Leonard, J. E, and C. A. Woolsey. The equivalence of controlled lagrangian and controlled hamiltonian systems. In ESAIM: Control, Optimization and Calculus of Variations, pages 393$422,2002$.

F. Gómez-Estern and Arjan J. van der Schaft. Physical damping in ida-pbc controlled underactuated mechanical systems. European Journal of Control, 10(5):451468, December 2004.

P. Kotyczka. Local linear dynamics assignment in ida-pbc for underactuated mechanical systems. In $C D C-E C C$, pages 6534-6539. IEEE, 2011.

P. Kotyczka and S. Delgado L. On a generalized porthamiltonian representation for the control of damped underactuated mechanical systems. In Proceedings 4 th IFAC Workshop on Lagrangian and Hamiltonian Methods for Non Linear Control, 2012.

R. Krechetnikov and J. E. Marsden. Dissipation-induced instabilities in finite dimensions. Rev. Mod. Phys., 79: 519-553, Apr 2007.

R. Ortega and Zhitao Liu. Control via interconnection and damping assignment of linear time-invariant systems: A tutorial. International Journal of Control, 2012.

R. Ortega and M. W. Spong. Stabilization of underactuated mechanical systems via interconnection and damping assignment. IEEE Trans. Aut. Control, 47: 1218-1233, 2000.

S. Prajna, A. Schaft van der, and G. Meinsma. An lmi approach to stabilization of linear port-controlled hamiltonian systems. Systems $\&$ Control Letters, 45(5): 371-385, April 2002.

M.W. Spong. Partial feedback linearization of underactuated mechanical systems. In Intelligent Robots and Systems '94. 'Advanced Robotic Systems and the Real World', IROS '94. Proc. of the IEEE/RSJ/GI Int. Conf. on, volume 1, pages $314-321$ vol.1, sep 1994.

C. Woolsey, A. M. Bloch, N. E. Leonard, C. K. Reddy, D. E. Chang, and J. E. Marsden. Controlled lagrangian systems with gyroscopic forcing and dissipation. European Journal of Control, 10(5):478-496, 2004.

D. V. Zenkov. Matching and stabilization of linear mechanical systems. Proc. MTNS. South Bend, IN., 2002. 